Fecha de recepción: marzo 2021 Fecha de aprobación: abril 2021 Fecha publicación: mayo 2021

\section{Prólogo. Guardar mientras se da. Reflexiones sobre las contribuciones teórico-metodológicas de Annette Weiner al estudio de las relaciones sociales mediadas por las cosas \\ Laura Colabella ${ }^{(1)}$ y Patricia Vargas ${ }^{(2)}$}

\begin{abstract}
Resumen: este Dossier homenajea a la antropóloga estadounidense Annette Weiner, una de las autoras pioneras en poner atención al papel relevante de las mujeres en los procesos de producción y reproducción social. La contribución de los participantes articula los conceptos centrales de la autora tributada con experiencias de investigación, cuyos trabajos de campo abordan la cultura material (en sus diferentes versiones). Museos y espacios de memoria, objetos de devoción, las casas y las cosas, los objetos que pueblan la vida cotidiana "puertas adentro" constituyen el protagonismo de aquello que se guarda, lo que se conserva, lo que se exhibe, lo que se hereda, lo que se regala.
\end{abstract}

Palabras claves: Weiner - Cultura Material - Teoría de la reciprocidad - Feminismos.

[Resúmenes en inglés y portugués en la página 21]

(1) Laura Colabella es Dra. en Antropología Social (MN-UFRJ), Profesora Asociada de Antropología, por la UNAJ y Profesora de la Maestría en Sociología y Ciencias Políticas de FLACSO- Argentina. Correo: m.laura.colabella@gmail.com

(2) Patricia Vargas es Dra. en Antropología Social (Universidad Iberoamericana, México), investigadora CAS-IDES/UNPAZ-IESCODE, Profesora Titular de Antropología en UBA XXI y en la Universidad de Belgrano, y Profesora Adjunta de Antropología en UNPAZ. Dicta cursos de métodos y trabajo de campo en programas de posgrado de Argentina y Chile. Correo: patriciabeatrizvargas@gmail.com

\title{
Introducción
}

Annette Weiner, una autora poco citada en la antropología argentina y, sin embargo, con una prolífera producción etnográfica en torno al intercambio, los roles de género, la producción de valor, los objetos y el consumo, representa sin dudas una figura que amerita ser reconocida por su potencialidad teórica y su compromiso con el trabajo de campo, una gran inspiración en estos tiempos. 
Nacida en Filadelfia, Pensilvania, estudió para técnica en radiología, se casó y tuvo dos hijas, antes de iniciarse en la disciplina antropológica. Su interés por las artes plásticas la acercó a la cultura material, sobre todo al mundo de los objetos. Dejando su casi exclusiva dedicación al ámbito doméstico, se inscribió en el Colegio para Mujeres de la Universidad de Pensilvania, y algunas lecturas antropológicas cambiaron su perspectiva, orientándose a la carrera de antropología.

En 1971 hizo trabajo de campo en las islas Trobriand, ampliando y profundizando el trabajo de Bronislaw Malinowski sobre la reciprocidad. Su análisis sobre "el ñame y los manojos de hojas de banana" producidos e intercambiados por mujeres, trastocó la atención que se dispensaba a la dimensión de género en la mantención del valor y el estatus e incentivó una nueva mirada para analizar los objetos, los ciclos de las cosas y el modo en que se inscriben dentro de diversas tramas de poder.

Como muy bien señalan en su recientemente publicado artículo Ohanian, Faccio y Blanco Esmoris, citamos:

El trabajo de investigación de la antropóloga Annette Barbara Cohen (19331997), mayormente conocida por su nombre de casada, Annette Weiner, siguió la dirección geográfica colonial propia de las academias del Norte hacia el sur (Guatemala, las Trobriand, Pakistán o Samoa) en los años setenta, en tres líneas de investigación: las teorías del intercambio, el análisis de los objetos y la cultura material, y las relaciones de género y el rol de las mujeres en los sistemas sociopolíticos, discutiendo desde el campo a los autores canonizados (Mauss y Malinowski).

Entre sus publicaciones se destacan "Mujeres de valor, hombres de renombre: nuevas perspectivas en el intercambio Trobriand" de 1976; "Los trobriandeses de Papúa Nueva Guinea" de 1988; “Posesiones inalienables" de 1992 y "La ropa / vestimenta y la experiencia humana" también de 1992, co-editado con Jane Schneider.

En esta introducción al dossier homenaje a la que denominamos la "antropóloga de los objetos", reflexionamos sobre tres cuestiones: la lectura de Maurice Godelier sobre las contribuciones de Weiner a la teoría de la reciprocidad; los encuentros y desencuentros con las perspectivas de Arjun Appadurai y de Igor Kopytoff, respecto al abordaje del estudio de las cosas cotidianas; y una conclusión de carácter metodológico que destaca el aporte analítico de nuestra autora tributada al campo de la cultura material. Asimismo, presentamos las contribuciones de cada uno de los diez artículos que componen este dossier, en diálogo con las ideas centrales de la autora tributada.

\section{Annette Weiner y la paradoja del don: una lectura desde Maurice Godelier}

El primer antecedente del Ensayo sobre el don es de 1910, una reseña de dos publicaciones de Swanton sobre el potlatch, donde refiere por primera vez al famoso "hecho social total". Mauss trabaja 15 años sobre el material de Franz Boas y sólo un año antes de mandar el 
Ensayo a prensa llega a sus manos el libro de Bronislaw Malinowski, sentando las bases para diferenciar intercambios agonísticos de no agonísticos. En el estudio preliminar de la versión española realizado por Fernando Giobellina Brumana, "El don del ensayo", el catedrático español nos advierte sobre la escasa repercusión de esta obra de Marcel Mauss, publicada entre 1923-1925, y la temprana crítica de Raymond Firth, alumno de Malinowski, sobre todo a la interpretación maussiana del hau.

Giobellina Brumana nos advierte que, no será sino hasta 1950 cuando, reimpreso como parte de una recopilación de sus textos ("Sociología y antropología") y con la introducción escrita por Lévi-Strauss, que la obra fuera reevaluada, y antropólogos y sociólogos destacados, como Marshall Sahlins, Edward Evans-Pritchard, Karl Polanyi, Maurice Godelier, Pierre Bourdieu y Maurice Merleu-Ponty citaran alguna parte del ensayo, consagrándolo como una referencia ineludible. Hasta ese momento Mauss ocupaba un lugar marginal en la academia, lejos de la Sorbona y la Escuela Normal Superior, y con una idea arcaica de la división del trabajo en la antropología, entre etnógrafos de campo y etnólogos de gabinete.

La antropóloga brasileña Lygia Sigaud introduce una complejidad novedosa a esta perspectiva, enfocándose en la relectura que esos antropólogos hicieron en los años cincuenta y sesenta, para compararla con la lectura de los contemporáneos de Mauss, en los años veinte. Para la autora, el Ensayo, es un texto sobre derecho, obligaciones y prestaciones totales (Sigaud, 1999, p. 89), preocupaciones del propio Mauss y sus contemporáneos al momento de su escritura. Sigaud retoma el análisis de las notas que le dieron origen al texto, para mostrar que las relecturas sólo recuperan la sección vinculada a los maoríes de la Polinesia, desconociendo el interés de Mauss por el potlatch kwakiutl. A su entender, la relectura académica de los años sesenta, operó una suerte de inflexión o discontinuidad con el sentido primigenio, que terminó por convertir al texto en un estudio sobre la teoría del intercambio; procedimiento que atribuye una fuerte identificación entre la cosa dada y el espíritu del dador, como principio de explicación. No en vano el Ensayo inicia preguntándose ¿Cuál es la regla de derecho y de interés que hace que, en las sociedades de tipo primitivo o arcaico, el presente recibido se devuelva obligatoriamente? ¿Qué fuerza hay en la cosa que se da que hace que el donatario la devuelva? (Mauss, 2009, p. 71).

Mauss aquí retoma para responder la teoría nativa maorí de Tamati Ranaipiri (interpretado por Mauss) como "lo que obliga en el regalo recibido, intercambiado, es el hecho de que la cosa recibida no es algo inerte": es el hau (que obliga a devolverlo). Y al profundizar en "la fuerza de las cosas" establece la distinción que nos llevará al aporte de nuestra antropóloga homenajeada:

Ante todo, al menos los kwakiutl y los tsimshian establecen entre los diversos tipos de sociedades la misma distinción que los romanos, los trobriandeses y los samoanos. Para ellos existen, por un lado, los objetos de consumo y de reparto vulgar, y por el otro, las cosas preciosas de la familia, los talismanes, los cobres blasonados, las mantas de pieles o de telas blasonadas (...)-transmitidos con solemnidad- en su caso no es exacto hablar de alienación. Son objetos de préstamo, más que de venta, y de verdaderas cesiones. Entre los kwakiutl, algunos de ellos, aunque estén presentes en el potlatch, no pueden ser cedidos. 
En el fondo, esas "propiedades" son sacra de las que la familia sólo se desprende con gran pena, y a veces, nunca (Mauss, 2009, pp. 167-169).

Dedica varias páginas a la descripción de este tipo de objetos preciosos (conchas de abalone, mantas blasonadas, fuentes, cucharas) que de alguna manera son, para Mauss, objetos animados, mágicos, con individualidad y valor propio, "bienes esenciales de muy estricta circulación y que se reparten con extremo cuidado entre los clanes y las familias de los jefes" para concluir que "las cosas tienen personalidad y las personalidades, de cierta forma, son cosas permanentes del clan” (Mauss, 2009, pp. 173-184).

Este párrafo del Ensayo es importante porque pone en foco la cuestión central de las clasificaciones nativas sobre las cosas, cómo deben circular, quién puede hacerlo, y también, cuáles deben ser guardadas, o de cuáles -como dice el mismo Mauss- la familia se desprende con gran pena.

Retomando este "enigma”, eje y nombre de su libro: El enigma del don (aquí utilizaremos la edición de 1998), el antropólogo francés Maurice Godelier atribuye a la lectura del Ensayo su interés por la antropología, y a la contribución de Weiner, la potencialidad de explicar por qué "hay cosas que los baruya (grupo humano con el que hizo su trabajo de campo) no podían ni vender ni donar, sino que debían guardar” (Ob. Cit, p. 19). Más aún, según sus propias palabras, a partir de la lectura de "Inalienable Possesions" surge su decisión de escribir un libro sobre el don. Ya en el título de la introducción nos advierte: "De las cosas que se dan, de las que se venden $y$ de las que no hay que dar ni vender, sino guardar", sintetizando su contribución al mencionar cómo Weiner (con su trabajo de campo realizado 50 años después que Malinowski) "había descubierto hechos nuevos que esclarecían problemas que Malinowski y Mauss habían dejado sin responder. Mostraba especialmente, cómo podía conservarse un objeto al mismo tiempo que se donaba, con lo que una parte del enigma del don quedaba así resuelta" (Ob. Cit, p. 20).

El enigma que propone Godelier, es dilucidar por qué se devuelve. Para Mauss, esto se produce porque las cosas donadas tienen un espíritu, que las obliga a retornar a la persona que en principio las ha poseído y donado (Ob. Cit, p. 29). Sin embargo, parte del enigma se devela al retomar justamente la distinción maussiana entre "el dominio de las cosas intercambiables, alienables, y el dominio de las cosas excluidas del intercambio, inalienables, que corresponden cada uno a los diferentes tipos de relaciones sociales y a diferentes momentos de la producción-reproducción de la sociedad" (Ob. Cit, p. 35).

Este Mauss “olvidado" para Godelier, es el que ofrece la clave a los aportes de Weiner: "no todas las riquezas se intercambian", siendo Mauss quien "distingue cuidadosamente dos categorías de objetos, los que pueden o deben donarse o intercambiarse, que son alienables, y los que no hay que donar ni intercambiar, en la medida en que son inalienables" (Ob. Cit, p. 53). Según Godelier, dos son los aportes centrales entonces de nuestra antropóloga tributada en este dossier: por un lado, la idea matriz de que:

Existen cosas que se deben guardar y no se deben donar. Esas cosas que se conservan (objetos preciosos, talismanes, saberes, ritos, etc.) afirman con precisión una serie de identidades y su continuidad en el tiempo. Más aún, afirman la existencia de diferencias de identidad entre los individuos, entre los grupos que 
componen una sociedad o que quieren situarse unos con relación a otros en el seno de un conjunto de sociedades vecinas conectadas entre sí por diversos tipos de intercambios. Pero esas diferencias de identidad no son neutras, sino que constituyen una jerarquía, y es en ese proceso de producción-reproducción de jerarquías entre individuos, entre grupos o incluso entre sociedades, donde ambas estrategias, el donar y el guardar, desempeñan roles distintos, aunque complementarios (...) de ahí su fórmula Keeping-while-Giving (Ob. Cit, pp. 54-55).

Para Godelier, la segunda idea fuerza de Weiner concierne a la "importancia de las mujeres y/o de lo femenino en el ejercicio del poder, en los mecanismos de legitimación y de redistribución del poder político-religioso entre los grupos que componen una sociedad" ya que muchos de los bienes preciosos estudiados por ella "son bienes femeninos, producidos por las mujeres y sobre los que disponen de derechos particulares" (Ob. Cit, p. 55).

Godelier discute a Weiner a la hora de pensar la relación entre estas esferas, que para él, se articulan a partir de su interdependencia y su diferenciación, por su autonomía relativa. Por eso, para él, la fórmula de lo social "no es Keeping-while-Giving sino Keeping-forGiving-for-Keeping. Guardar para (poder) donar, donar para (poder) guardar” (Ob. Cit, p. 58). Desde su trabajo de campo, concluye que, entre los baruya, la cosa donada no es alienada porque quien la dona continúa conservando los derechos sobre lo donado.

Retomando el legado de Mauss, dice Godelier respecto de las riquezas inalienables que se trata de "cosas sagradas que se transmiten de generación en generación por medio del matrimonio y a través de la herencia", son más bien objetos de préstamo o cesión, producen y atraen riquezas porque son "divinas", "dones que espíritus o dioses hicieron a los humanos, espíritus o dioses que continúan estando presentes en ellas, actuando sobre quienes las poseen en la actualidad, por haberlas recibido de sus antepasados o del héroe fundador del clan, quien a su vez las recibió de un espíritu" (Ob. Cit, pp. 91-92).

Mauss debería haberse preguntado

¿Por qué entre todos esos objetos que tienen un alma, unos, los más numerosos, pueden donarse y circular entre los individuos y los grupos 'sin llegar a separarse del todo' de sus propietarios originales, sin alienarse completamente, mientras que otros, más preciosos, más sagrados, no circulan y permanecen inmóviles en los tesoros de los clanes y las familias? (...) la división de la sociedad en subgrupos 'imbricados unos en otros' que 'se deben todo', no implica en modo alguno que se donen todo (Ob. Cit, p. 103).

Es así como le reconoce a Weiner la importancia de señalar cómo (en el kula) "Mientras circula, el objeto es a la vez donado y guardado, se guarda la propiedad del objeto y se dona su posesión, que se va transfiriendo de asociado intermediario a asociado intermediario" (Ob. Cit, pp. 134-135). Al respecto, Godelier sostiene que "no es el objeto el que crea las diferencias, sino las distintas lógicas de los dominios de la vida social quienes le confieren sentidos distintos a medida que se desplaza de uno a otro, cambiando con ello de función y de uso" (Ob. Cit, p. 157), concluyendo que ninguna sociedad humana podría existir sin 
estos dos dominios, el de los intercambios y el de la conservación, donde los individuos y grupos guardan preciosamente para sí cosas, relatos, nombres y formas de pensamiento, que posteriormente transmiten a sus descendientes, o a quienes comparten su misma fe.

\section{Encuentros y dispersiones con la vida social de las cosas de Arjun Appadurai y la biografía de los objetos de Igor Kopytoff}

Una segunda discusión interesante a la hora de pensar la cultura material y en particular cómo estudiar antropológicamente a los objetos, es preguntarnos sobre los sistemas de clasificación de los interlocutores de trabajo de campo y las contribuciones de Annette Weiner, sobre la "creación de valor" a través de los procesos de "densificación simbólica de las cosas", y comparar estas ideas con la estructura conceptual y metodológica que proponen Arjun Appadurai, en su introducción al libro de 1986, La vida social de las cosas. Perspectiva cultural de las mercancías, y la propuesta de Igor Kopytoff sobre La biografía cultural de las cosas: la mercantilización como proceso. Entre ambas perspectivas podemos hallar, justamente, encuentros y dispersiones.

La preocupación central de Weiner es acerca de la creación de valor en objetos que permanecen fuera de circulación (se guardan): "la inalienabilidad se expresa a través del poder que tienen los objetos para definir quién es quién en un sentido histórico". Ella examina la importancia de diferentes formas de riqueza (paquetes de hojas secas de plátano, esteras y capas) imbuidas de un valor sagrado a partir de su hasta 200 años de conservación y exhibición en momentos rituales (bautizos, casamientos o funerales), y que, al retenerlos, mantienen, documentan y consolidan la identidad ancestral del clan, reproduciendo relaciones y jerarquías (Weiner, 1985). El valor se crea al sustraer ciertas posesiones del proceso de intercambio, produciendo "densidad simbólica", manteniéndolas en el contexto cerrado de la familia o del clan. Entre estas "posesiones inalienables" podemos encontrar, según Weiner, aquellas que "nunca deberían circular" y aquellas que, bajo ciertas circunstancias, "se pueden dar en calidad de préstamos, como copias o a cambio de otro objeto del mismo tipo o equivalente"; pero más que los objetos lo que importa es quién es el dueño y qué papel cumple en la creación o legitimación de diferencias sociales. Transferir estos objetos conservados, a personas aprobadas para perpetuar el legado, oficia de estabilizador de las identidades a través del tiempo (Weiner, 1994).

Estos postulados teóricos que surgen de su trabajo de campo, focalizan en sociedades de pequeña escala, con economías preindustriales o en procesos de descolonización. Sin embargo, la misma Weiner se interroga respecto a cómo el contexto capitalista podría afectar la inalienabilidad, y es aquí donde se pueden establecer dos puentes con las reflexiones en torno a las cosas (y su mercantilización) en diálogo con Appadurai, en un enfoque más macro-estructural y con Kopytoff, en un sentido más micro-individual.

Appadurai propone que "la situación mercantil de las cosas se vincula a su intercambiabilidad", es decir que "pueden entrar y salir del estado mercantil". La pregunta es entonces, un objeto culturalmente significado como inalienable ¿puede transformarse en mercancía? O en términos de dicho autor ¿en cierta fase de su trayectoria y en un contexto parti- 
cular, cubre los requisitos de la candidatura mercantil? Al igual que Godelier, Appadurai acuerda con la idea de que no es el tipo de objeto el que determina que sea una mercancía o no, sino que la mercantilización es una fase potencial en la que, mediaciones culturales socio-históricas de por medio, puede ingresar cualquier tipo de objeto. El mismo Appadurai menciona en esta célebre introducción los aportes de Weiner para repensar la política de la creación de valor y de producción de inalienabilidad, justamente a partir de la idea de enclaves, toda vez que pueden institucionalizarse, extraer y proteger a los objetos contra los contextos mercantiles socialmente relevantes, y de desviaciones, que aspiran a arrastrar las cosas protegidas a la zona de la mercantilización (por ejemplo, penurias económicas, guerras o saqueos). Para Appadurai, las desviaciones sólo pueden entenderse en su relación dialéctica y constitutiva con respecto a las rutas de las cuales se apartan, es decir, cómo se podría alienar lo inalienable.

Lo que Appadurai presenta para pensar los grandes procesos en general -la historia social de las cosas-, Kopytoff lo aplica a procesos específicos -la biografía cultural de los objetos-. Para este autor "la cultura asegura que algunas cosas permanezcan inequívocamente singulares, evita la mercantilización de otras y, en ocasiones, resingulariza lo que ha sido mercantilizado" (Kopytoff, 1986, p. 100). Lo sagrado, lo simbólicamente denso y la relación temporal y sentimental que une ciertos objetos a las personas pueden volver culturalmente impensada su mercantilización: a esto que Kopytoff denomina "singularización" podríamos llamar en términos de Weiner, "inalienabilidad", aunque se trate de lo profano y lo cotidiano, y siempre exprese un carácter potencialmente ambiguo. Como dice Kopytoff:

En el mundo homogeneizado de las mercancías, la biografía memorable de una cosa se convierte en la historia de las diversas singularizaciones experimentadas por ella, de las clasificaciones y reclasificaciones a las cuales ha estado sujeta en un mundo incierto de categorías cuya importancia varía con cada modificación menor del contexto. Al igual que en el caso de las personas, el drama reside aquí en la incertidumbre tanto de la valoración como de la identidad (Kopytoff, 1986, pp. 119-120).

\section{Contribuciones al presente dossier}

Este dossier tuvo como antecedentes dos eventos académicos realizados virtualmente en julio y agosto de 2020 respectivamente. Por un lado, el XI Congreso Latinoamericano de Enseñanza del Diseño organizado por la Universidad de Palermo; por otro, las IX Jornadas de Etnografía y Métodos Cualitativos, organizadas por el Centro de Antropología Social del Instituto de Desarrollo Económico y Social (CAS-IDES). En ambos eventos, Laura Colabella y Patricia Vargas, coordinaron mesas y simposios homenaje a la antropóloga Annette Weiner, donde se discutieron los trabajos que aquí se presentan. También contamos con la presencia del Grupo de Estudio y Trabajo sobre Cosas Cotidianas (cultura material) (CAS-IDES) y del Equipo Multidisciplinario de Investigación Etnográfica sobre Cultura 
Material en José Clemente Paz, con sede en el Instituto de Estudios Sociales en Contextos de Desigualdades de la Universidad Nacional de José C. Paz (IESCODE-UNPAZ).

Retomando los intercambios construidos en ambos espacios académicos, hoy celebramos la presentación de esta publicación colectiva que se inscribe en la línea de investigación "Etnografía, cultura material y educación. Aportes desde las perspectivas de Pierre Bourdieu y Annette Weiner", desarrollada en la Facultad de Diseño y Comunicación de la Universidad de Palermo.

El texto que inaugura este Dossier corresponde a Gabriel Noel quien a partir del reexamen de la evidencia etnográfica y de la minuciosa presentación y análisis de Weiner con relación a las mismas, nos invita a pensar acerca del lugar de la descripción y comparación en las publicaciones científicas actuales y en los modos contemporáneos de producción, circulación y publicación en la disciplina antropológica

Seguidamente, los trabajos de Jazmín Ohanian y Ana Gugliemucci nos acercan, desde Weiner, a un tema más que sensible para la Argentina y Colombia como es la memoria social. El primero de ellos, nos lleva a la Escuela Mecánica de la Armada (ESMA), cuando un grupo de ex alumnos condujeron a la investigadora al sótano del Círculo de Oficiales de Mar donde habían curado un "museo" exclusivo para personal de la Armada y donde narraban la historia de la institución educativa a través de la exhibición de objetos (entre ellos: boletines escolares, tierra, restos de papelitos, platos y carnets). Según Ohanian, éstos "conservan y muestran la fuerza invertida por ellos en resguardar objetos que pasaron de ser cotidianos e intercambiables a ser inalienables". El trabajo de Gugliemucci, por su parte, nos muestra el tratamiento concedido a los objetos que pertenecieron a personas desaparecidas (fotografías, vestimenta, cartas, diarios íntimos y juguetes) en Argentina y Colombia. Más precisamente, en movilizaciones públicas y obras artísticas como la Obra Relicarios. Para la autora, lo que antes podía ser considerado como una mercancía, en un mundo caracterizado por la intercambiabilidad y la obsolescencia programada de casi todas las cosas, suele transformarse en un objeto intransferible o exclusivo a raíz de la ausencia forzada de su poseedor; tornando ese bien en una posesión inalienable en la perspectiva de Weiner.

A continuación, los trabajos de Noelia Lopez y Paola Castro Molina, nos llevan a escenarios rurales donde la agricultura es la actividad principal cercada por la religiosidad y la migración. Lopez nos lleva al conurbano sur, concretamente al municipio de Florencio Varela, en cuyos confines se encuentran las fincas productoras de las principales verduras que se consumen en la ciudad de Buenos Aires; y que están, en su mayoría, gerenciadas por migrantes bolivianas. La autora mostrará cómo al momento de la cosecha, cuando los cultivos estaban listos para levantar, es decir, destinados a la comercialización; uno de ellos - el maicito de Bolivia - recibía un tratamiento diferencial al ser excluido del circuito comercial y ubicándolo, de esa manera, como un posible objeto inalienable.

Por su parte, Castro Molina nos lleva al municipio de Puna, del departamento de Potosí en Bolivia, durante la celebración de las Pascuas. Esta festividad, a pesar de ser paralela a la Semana Santa católica, es más bien un ritual de petición de lluvia para fines agrícolas donde la presencia de los cajones religiosos (objetos de madera que exhiben imágenes religiosas pintadas sobre piedras o modeladas en yeso, en las respectivas capillas) son reverenciadas por los participantes al quitarse el sombrero y rezarles. Para la autora, estos cajones 
equivalen a las inalienable possessions de Weiner por el cuidado y resguardo concedido a los mismos, antes y después de la celebración.

Continúa en la presentación, el trabajo de Luciana Denardi. Se trata del único texto del dossier que aborda el mundo de los negocios, a partir de una categoría nativa (Guānxi) que alude tanto a la capacidad de construir lazos sociales como al intercambio de favores, regalos, creación de obligaciones y deudas. La autora mostrará las escenas en que el Guānxi se hizo presente en su trabajo de campo con migrantes chinos en Buenos Aires. Siguiendo a Weiner, Denardi mostrará cómo para comprender esa red por la que circulan diferentes objetos alineables (dinero, regalos, banquetes) e inalienables (confianza, amistad), es necesario inscribirla en un sistema más amplio basado en un trabajo relacional continuo que responde a valores confucianos.

Dándole continuidad a los trabajos sobre el gran Buenos Aires, María Florencia Blanco Esmoris analiza el sentido del habitar de Luisa, una odontóloga residente en Morón, Provincia de Buenos Aires, quien intercambia la casa familiar con su hijo, para mudarse a un $\mathrm{PH}, \mathrm{y}$ darle un nuevo sentido de realización a su vida. A partir de la creación de una categoría interpretativa, la regeneración afectivo-habitacional, la autora retoma la contribución de Weiner sobre los procesos de regeneración femeninos, donde se conjugan los ciclos vitales y los recursos materiales y simbólicos, poniendo el foco en los objetos que entran, salen y se intercambian en dichos procesos.

Puertas adentro, encontramos un mundo de "objetos inalienables" en las viviendas de cinco estudiantes de la Universidad Nacional de José Clemente Paz, ubicada en el partido homónimo del Conurbano. Florencia Blanco Esmoris, Martina Cassiau, Julieta Concilio, Facundo Finamore, Julieta Impemba, Melanie Liberman, Lorena Schiava D’Albano y Patricia Vargas asumen el desafío de escribir un artículo conjunto, producto de una propuesta de etnografía colectiva. Un terreno familiar, un Gauchito Gil que vino con la casa, una escultura devocional del Divino Niño, una colección de mates y cuchillos y un modular, son las cosas a través de las cuales sus propietarios expresan una relación particular con dichas posesiones, poniendo en evidencia cómo se consolidan, transforman o rompen, los lazos sociales (constituyéndose mutuamente).

Martina Cassiau, por su parte, nos lleva con una pareja de Capital hasta Catamarca, a conocer a las famosas bordadoras de acolchados, poseedoras de saberes ancestrales. Colchas y saberes, se guardan o se ponen en circulación, según los contextos, las urgencias o las oportunidades. Como en el caso analizado por Weiner, en un contexto de escasez, los mantones se transforman en "objetos valiosos que producen jerarquía, prestigio y status" en la pequeña comunidad local, consolidando no sólo la diferencia sino también viejas rivalidades y profundizando las desigualdades.

Y, por último, volvemos a Capital con Lorena Schiava D'Albano, quien nos ubica en el corazón de Recoleta, para pensar en el proceso de producción de inalienabilidad en el marco del capitalismo globalizado. Realzando el contrapunto entre el trabajo de campo de Weiner, en pequeñas aldeas, y su propio análisis de los "objetos de moda icónicos" que conforman la currícula de la carrera de Asesor de Imagen, esta autora nos invita a pensar ¿por qué tenemos que llegar a tener zapatos Ferragamo o carteras Hermès para ser un determinado tipo de persona?, y a mirar de cerca el derrotero de dichos objetos, su adquisición, conservación y transferibilidad. 


\section{Reflexiones finales: el valor del trabajo de campo en el abordaje de la cultura material y el mundo de las cosas cotidianas}

Según inicia su introducción la misma Weiner en su libro seminal:

Caminar por una aldea al comenzar un trabajo de campo es ingresar a un mundo sin referencias culturales. La tarea de aprender los valores que otros viven nunca es fácil. Los rigores del trabajo de campo incluyen escuchar y mirar, aprender un nuevo lenguaje de palabras y acciones, $y$, antes que nada, permitir apartarse de los propios supuestos culturales con el objeto de comprender los sentidos que otros dan al trabajo, el poder, la familia y los amigos.

Y eso es lo que ella hace cuando reconoce la centralidad del trabajo de campo como ejercicio central del oficio antropológico.

Ella misma expresa que la diferenciación con el análisis de Malinowski fue la atención dispensada al trabajo productivo de las mujeres, una atención que no estaba planificada por la investigadora que quería estudiar a los famosos talladores hombres de la zona. Sin embargo, las mujeres llevaron a Weiner a observar una forma de distribución de sus propias riquezas -fardos de hojas de banana y polleras de fibras de banana- que intercambiaban con otras mujeres en un contexto funerario. "Nuestros fardos son como tu dinero", le espetaron, obligándola a considerar los roles económicos femeninos y poniendo en valor la contribución de las mujeres en los procesos de producción y reproducción social.

Casi 50 años después de este incidente de campo, reafirmamos la potencia del detalle etnográfico y su fuerza para derrotar al tiempo; la humildad para reconocer los aportes de nuestros predecesores tanto sobre los temas que trabajamos como sobre los espacios sociales y casos que queremos comprender, la pasión para mantener el asombro que produce el aprendizaje de lo que es relevante para los Otros, en sus propios términos y la flexibilidad para cambiar, como hizo Weiner, a los famosos talladores que ganaban dinero por las acciones aparentemente anodinas de estas mujeres que le ayudaron a crear contribuciones inalienables para la antropología contemporánea.

\section{Bibliografía}

Appadurai, A. (1986). Introducción: Las mercancías y la política del valor en Appadurai, A. (comp.) La vida social de las cosas. Perspectiva cultural de las mercancías. México: Grijalbo. Godelier, M. (1998). El enigma del don. Barcelona, Paidós.

Kopytoff, I. (1986). La biografía cultural de las cosas. La mercantilización como proceso. En Appadurai, A. (comp.) La vida social de las cosas. Perspectiva cultural de las mercancías. México: Grijalbo.

Mauss, M. (2009). Ensayo sobre el don. Forma y función del intercambio en las sociedades arcaicas. Buenos Aires: Katz Editores. 
Ohanian, M. J.; Faccio, Y. y Blanco Esmoris, M. F. (2020). Annette B. Cohen/Weiner: notas sobre una trayectoria antropológica singular, en Cuadernos de Antropología 30 (2), 1-15. Julio a diciembre 2020.

Sigaud, L. (1999). As vicissitudes do Ensaio sobre o dom, en Mana 5 (2):89-124.

Weiner, A. (1985). Inalienable wealth, en American Ethnologist,Vol. 12, № 2 (May, 1985), pp. 210-227.

Weiner, A. (1988). The Trobrianders of Papua New Guinea. New York: Holt, Rinehart and Winston.

Weiner, A. (1992). Inalienable possessions. The Paradox of Keeping-While-Giving. California: University of California Press.

Weiner, A. (1994). Cultural difference and the density of objects, en American Ethnologist, Vol. 21, No 2 (May, 1994), pp. 391-403.

\begin{abstract}
This dossier pays tribute to the American anthropologist Annette Weiner, one of the pioneering authors in paying attention to the relevant role of women in the processes of social production and reproduction. The contribution of the participants articulates the central concepts of the taxed author with research experiences, whose field work addresses material culture (in its different versions). Museums and memory spaces, objects of devotion, houses and things, objects that populate daily life "indoors" are the protagonists of what is kept, what is preserved, what is exhibited, what is inherited, what is given away.
\end{abstract}

Keywords: Weiner - Material Culture - Reciprocity Theory - Feminisms.

Resumo: Este dossiê homenageia a antropóloga americana Annette Weiner, uma das autoras pioneiras em dar atenção ao relevante papel da mulher nos processos de produção e reprodução social. A contribuição dos participantes articula os conceitos centrais do tributado autor com experiências de pesquisa, cujo trabalho de campo aborda a cultura material (em suas diferentes versões). Museus e espaços de memória, objetos de devoção, casas e coisas, os objetos que povoam o cotidiano "dentro de casa" são os protagonistas do que se guarda, do que se conserva, do que se exibe, do que se herda, do que se doa.

Palavras chave: Weiner - Cultura material - Reciprocidade - Feminismos.

[Las traducciones de los abstracts fueron supervisadas por el autor de cada artículo] 